\title{
Analyzing Forensic Evidence Based on Density with Magnetic Levitation
}

\section{Citation}

Lockett, Matthew R., Katherine A. Mirica, Charles R. Mace, Robert D. Blackledge, and George M. Whitesides. 2013. Analyzing Forensic Evidence Based on Density with Magnetic Levitation. Journal of Forensic Sciences 58, no. 1: 40-45.

\section{Published Version}

doi:10.1111/j.1556-4029.2012.02221.x

\section{Permanent link}

http://nrs.harvard.edu/urn-3:HUL.InstRepos:11928052

\section{Terms of Use}

This article was downloaded from Harvard University's DASH repository, and is made available under the terms and conditions applicable to Open Access Policy Articles, as set forth at http:// nrs.harvard.edu/urn-3:HUL.InstRepos:dash.current.terms-of-use\#OAP

\section{Share Your Story}

The Harvard community has made this article openly available.

Please share how this access benefits you. Submit a story.

Accessibility 


\section{Analyzing Forensic Evidence Based on}

\section{Density with Magnetic Levitation ${ }^{\dagger}$}

Matthew R. Lockett ${ }^{1}$, Katherine A. Mirica ${ }^{1}$, Charles R. Mace ${ }^{1}$, Robert Blackledge ${ }^{2 *}$, and George M. Whitesides ${ }^{1,3 *}$

${ }^{1}$ Department of Chemistry \& Chemical Biology, Harvard University, Cambridge, MA 02138

${ }^{2}$ El Cajon, CA 92021

${ }^{3}$ Wyss Institute for Biologically Inspired Engineering, Harvard University, Cambridge, MA 02138

*Corresponding author E-mail: gwhitesides@gmwgroup.harvard.edu, bigpurple@cox.net

${ }^{\dagger}$ This work was supported by the Bill and Melinda Gates Foundation award number 57308 and the Wyss Institute for Biologically Inspired Engineering. 


\begin{abstract}
:
This paper describes a method for determining the density of contact trace objects with magnetic levitation. The density of samples of glitter and of gunpowder was determined, and the feasibility of magnetic levitation as a possible means of characterizing forensic-related evidence is discussed. The magnetic levitation device (composed of two permanent magnets with like poles facing) and the method described provides a means of accurately determining the density of trace objects that is inexpensive, rapid, verifiable, provides documentation, is independent of the specific apparatus or analyst, and provides numerical values (rather than a comparison between questioned and known samples) that may be entered into a searchable database.
\end{abstract}


Common contact trace objects are used in forensic investigations to establish an association (i.e., link criminals to crime scenes and to victims). Most of these objects interact weakly with a magnetic field (i.e., are diamagnetic in nature) - hairs, fibers, paint chips, and fragments of broken glass. Glitter is another - less often exploited - contact trace material that has been used as associative evidence. ${ }^{[1]}$ Contact trace objects are characterized with tests that range in difficulty and expense, from visual or microscopic inspection to spectroscopic analysis. The density of an object is another physical parameter that could, in principle, be used to characterize a trace object. Measuring the density of an irregularly-shaped object requires a displacement measurement to determine its volume. Volume measurements are difficult for small objects such as glitter particles, and thus there exists a need for a method that makes it possible to determine the density of trace objects quickly and easily. Kirk developed a method for analyzing glass fragments that was both comparative and could be assigned a numerical density value. ${ }^{[2]}$ This method, often called the "sink/float method," uses density columns that are prepared by mixing two organic liquids (e.g., bromoform and bromobenzene) in a specific ratio. Questioned and known glass fragments are placed together in the mixture, and the ratio of the organic liquids is adjusted until the two glass fragments could be distinguished (i.e., one fragment floated to the top of the density column while the other sank to the bottom). If the two glass fragments could be separated then the glass samples could not have originated from the same source. If the two glass fragments could not be separated (i.e., floated or sank together) and could also be suspended within the density column at a specific liquid ratio, then one could conclude both fragments originated from the same source, and that their density corresponded to the density of the liquid mixture. 
Magnetic levitation ("MagLev") of diamagnetic objects is a new method of determining density that is well-suited for analyzing contact trace objects: i) it does not destroy the sample, ii) it is readily calibrated with a series of density standards, and iii) it is applicable to small and irregularly shaped particles. MagLev is a technique whose sensitivity can be adjusted to the application. With care, MagLev can distinguish between two diamagnetic objects whose densities differ by $0.0002 \mathrm{~g} / \mathrm{cm}^{3} \cdot{ }^{[3]}$ This technique involves placing diamagnetic samples into a container filled with a paramagnetic fluid, which is then placed between two permanent magnets. The vertical position of the sample, in the presence of the magnetic field, correlates with its density. This position of the sample is independent of mass or volume separately, and measurements of density by MagLev thus do not require standardized sample sizes. ${ }^{[3,4]}$ The MagLev device we use ( 8 $\mathrm{cm} \times 6 \mathrm{~cm} \times 12 \mathrm{~cm}$ ) is both portable and inexpensive to fabricate (the NdFeB magnets cost $\sim \$ 20$ each), ${ }^{[3]}$ and does not require additional external equipment. A MagLev-based density measurement can be obtained in a short period of time (seconds to minutes, depending on the size of the object).

We used MagLev to determine the density of glitter samples of known density, glitter samples of unknown density, and smokeless gunpowder samples of unknown density. We determined that prolonged exposure to a solution of $\mathrm{MnCl}_{2}$ (i.e., the paramagnetic solution used in our experiments) does not affect the density of a sample of smokeless gunpowder. We also determined that repeated measurements (i.e., repeated exposure to a solution of $\mathrm{MnCl}_{2}$ and washing and drying steps) do not affect the density of a sample of smokeless gunpowder.

\section{Experimental Design.}




\section{MagLev Device.}

We establish the magnetic field in the MagLev system by aligning two NdFeB magnets $(5 \mathrm{~cm} \times 5 \mathrm{~cm} \times 2.5 \mathrm{~cm})$ co-axially, $4.5 \mathrm{~cm}$ apart, with like poles facing each other. The relative position of an object in the vertical direction when placed in the MagLev device, its "levitation height", is reached when the gravitational $\left(\mathrm{F}_{\mathrm{g}}\right)$ and magnetic forces $\left(\mathrm{F}_{\mathrm{m}}\right)$ acting,${ }^{[3]}$ in opposite directions, on the object have the same magnitude ${ }^{[3]}$ We define the levitation height as "h" in Figure 1. This position correlates, linearly, with the density of the sample. The analytical expression (and the associated assumptions and approximation) for correlating the levitation height of the sample with their density are described elsewhere.$^{[3]}$ A detailed knowledge of the parameters involved in making this correlation (e.g., the density of the paramagnetic medium, magnetic susceptibility of the medium, the magnetic field at the surface of the magnets) or a comprehensive understanding of the physics of MagLev are not necessary and can by disregarded when density measurements are made with standards of known density.

\section{Choice of Analytes.}

The objects commonly encountered in crime scenes (smokeless gunpowder, fibers, hair, glitter, etc.) are diamagnetic, and thus interact weakly with an applied magnetic field. MagLev of these samples is achieved by placing them in a solution containing a strongly paramagnetic ion. In a magnetic field gradient, suspended in a paramagnetic fluid medium, diamagnetic samples appear to be repelled from regions of high magnetic field; in actuality, the diamagnetic object displaces an equal volume of paramagnetic solution, and it is the attractive interaction between this paramagnetic volume and the regions of high magnetic field and this paramagnetic volume that results 
in MagLev. We and others have shown that a variety of aqueous solutions of paramagnetic salts (e.g., $\mathrm{MnCl}_{2}, \mathrm{MnSO}_{4}, \mathrm{GdCl}_{3}, \mathrm{FeCl}_{3}, \mathrm{CuSO}_{4}$, etc.) and chelated paramagnetic ions (e.g., Gd(DTPA) and Mn(EDTA); both in aqueous and non-aqueous solutions) are suitable for MagLev. ${ }^{[3-6]}$

\section{Operating Procedure.}

In the MagLev experiments presented here, we placed each sample in a $1 \mathrm{~cm} \times 1$ $\mathrm{cm} \times 4.5 \mathrm{~cm}$ cuvette containing $\mathrm{MnCl}_{2}$ (chosen because the solutions are transparent and because manganese salts are inexpensive), capped the cuvette, and then inverted the cuvette several times to remove any bubbles that may have formed on the samples. We explored the benefits of using a degassed $\mathrm{MnCl}_{2}$ solution, of adding a small amount of detergent (Tween 20, $0.1 \%(\mathrm{vol} / \mathrm{vol})$ ) to the $\mathrm{MnCl}_{2}$ solution, and sonicating the sample when preparing the glitter samples. The addition of detergent lowers the surface tension of the aqueous paramagnetic medium, and reduced interactions between hydrophobic objects, as well as their interactions with the walls of the cuvette. Bubbles that form on the walls of the cuvette and on the sample itself can be removed with sonication or by using a sample that has been degassed (e.g., bubbles present on the sample are absorbed into the degassed solution). The presence of a bubble on a sample can greatly influence its overall density (e.g., the density of air is $0.00118 \mathrm{~g} / \mathrm{cm}^{3}$ at $25^{\circ} \mathrm{C}$ at standard pressure). ${ }^{[7]}$

We placed the cuvette between the magnets, and took a photograph once the samples reached a stable levitation height ( 3 minutes, Figure 2$)$. We used ImageJ, a freeware image-processing package available from the $\mathrm{NIH}^{[8]}$, to determine the levitation 
height of each sample. Each photograph contained a ruler with millimeter markings, which we used as a reference when determining levitation height values. To analyze each image: we imported the color photograph into ImageJ and converted it into a gray scale image; we then adjusted the contrast using the "auto contrast" function and had the software outline the areas of high contrast with the "find edges" function; we then measured the distance from the bottom of the cuvette to the center of the outlined object. We compared the levitation height determined from the ImageJ with that of the ruler in the photograph. We used density standard beads (purchased from American Density Materials Inc., Staunton, VA) to calibrate each measurement. The accuracy of each standard was $\pm 0.0002 \mathrm{~g} / \mathrm{cm}^{3}$. Polymers, of known density, may also be used in MagLev experiments as a density standard.

Glitter Samples.

Glitter is a synthetic product, whose composition can range from tiny pieces of aluminum foil to multiple layers of plastic with (or without) an applied metal layer. ${ }^{[9]}$ Glitter is, in many ways, an ideal contact trace: it is nearly invisible; has a high probability of transfer and retention; is highly individualistic; can be quickly collected and separated; small traces are easily characterized; and measured properties of the glitter particles can be placed into a searchable database. ${ }^{[9,10]}$ Glitter particles can be characterized by a large number of methods: i) Visual inspection of color, shape, size, and morphology. This approach is problematic, however, as many glitter pieces do not contain dyes or pigments, or have "color shifting" effects that vary with lighting and the angle of observation. ii) The thickness of the glitter particles can be measured mechanically or spectroscopically. iii) Attenuated total reflection (ATR) FTIR and 
Raman microspectroscopy provide insight into the chemical structure of the polymers and coatings used in glitter production. ${ }^{[9]}$

Glitter particles, from a commercial vendor, have density values ranging from 1.2 $-2.5 \mathrm{~g} / \mathrm{cm}^{3} .^{[11]}$ Density measurements of glitter particles could provide valuable insight when: i) Two glitter pieces that have a similar shape and color have different densities. ii) Two pieces of glitter are composed of the same polymer base but differ in density because of the materials applied to them (organic dyes, polymeric materials, and metal particles). iii) The heterogeneity of a glitter sample may also be determined from MagLev measurements, based on the range of observed levitation heights.

\section{Smokeless Gun Powder Samples.}

Smokeless powders are a class of explosive propellant, consisting of gelatinized nitrocellulose with double base powders containing nitroglycerin, that produce very little smoke upon deflagration. ${ }^{[12]}$ The decomposition of each of these compounds, results in the release of nitrates, and requires that a stabilizer be added, typically diphenylamine. ${ }^{[13]}$ The ratio of each of these materials, as well as methods of batch preparation, result in density differences in smokeless gunpowder samples/batches. Smokeless powders are encountered in forensic science in the form of residues from gunshots or explosive devices. ${ }^{[14]}$ The shape and size of the powder particles have marked differences on the burning rate and power generation, with many powders being disk-, cylinder-, or ballshaped. There are a number of analytical techniques that are used to narrow the number of brands of smokeless powders to one or a few; this process begins with morphological characterization and proceeds to other types of physical and chemical characteristics. ${ }^{[15]}$ Analysis of MagLev Data. 
We prepared stock solutions of $\mathrm{MnCl}_{2}$ and used them throughout the

experiments. The use of density standard beads ensured that minor changes in the relative $\mathrm{MnCl}_{2}$ concentration were accounted for. The Results and Discussion section provides detailed information of the concentrations of $\mathrm{MnCl}_{2}$ and the density of the standards used in each experiment.

We made seven measurements of levitation height for each sample. We measured the distance from the surface of the bottom magnet to the vertical midpoint of each object to determine its levitation height. In a single measurement of levitation height, we placed three pieces of gunpowder or $10-20$ pieces of glitter in the appropriate $\mathrm{MnCl}_{2}$ solution. ${ }^{[16]}$ We placed a ruler, with millimeter markings, next to the cuvette containing the sample to make preliminary measurements of height and to aid in making height relationships by eye. We measured the levitation height of each object with the ImageJ software package. We determined the average height of the glitter (or gunpowder) samples, as well as their deviation from the mean, for each MagLev measurement. The standard deviations reported in this work were calculated with standard error propagation methods, ${ }^{[17]}$ and account for each MagLev measurement.

\section{Results and Discussion.}

\section{MagLev as an Analytical Tool in Forensic Science.}

We measured the density of eleven samples of glitter, each of which was silver in color, by MagLev. We obtained glitter samples from Meadowbrook Inventions and Table 1 summarizes their properties. We levitated each sample of glitter in an aqueous solution of 3.0M $\mathrm{MnCl}_{2}$; we used density standard beads with densities of 1.350 and $1.450 \mathrm{~g} / \mathrm{cm}^{3}$ to extrapolate the density of each piece of glitter. Figure 3a contains images of Crystalina 
\#321 (Table 1) at 0 and 360 seconds after the cuvette of glitter was placed in the MagLev device. We waited for approximately three minutes before obtaining photographs of the glitter and gunpowder samples, based on the time series photos obtained in Figure 2.

The density of each glitter sample was measured using MagLev, with a precision of $\pm 0.001 \mathrm{~g} / \mathrm{cm}^{3}$, and compared with the density values provided by the manufacturer. The relative standard deviation for each glitter sample was less than 3.0\%.

The measured densities for the eleven glitter samples can be broken down into three categories, when compared to the density values reported by the manufacturer: in high agreement with an average density difference of less than $0.05 \mathrm{~g} / \mathrm{cm}^{3}$, moderate agreement with an average density difference of greater than $0.05 \mathrm{~g} / \mathrm{cm}^{3}$ but less than $0.10 \mathrm{~g} / \mathrm{cm}^{3}$, and low agreement with an average density difference of greater than 0.10 $\mathrm{g} / \mathrm{cm}^{3}$. Seven samples were in high agreement with the reported density values, two samples were in moderate agreement, and two samples differed by more than $1.0 \mathrm{~g} / \mathrm{cm}^{3}$ from the reported density values (Alpha Jewels I and II). It should be noted that each of the density values measured by MagLev are less than those reported by the manufacturer, however when rounded to an equivalent number of significant figures six of the eleven samples match.

We determined the density of a glitter sample, of unknown density, from commercial nail polish. We separated the glitter particles from the polish by dissolving $0.5 \mathrm{~g}$ of the product in $5 \mathrm{~mL}$ of acetone, which we then collected by passing the solution through a piece of Whatman 1 quantitative filter paper. A similar procedure for extracting shimmer particles from make up samples has been reported by Griggs et al. ${ }^{[18]}$ The density of the glitter contained in "New York Color starry silver glitter" $(1.274 \pm 0.034$ 
$\left.\mathrm{g} / \mathrm{cm}^{3}\right)$ and "Sally Hansen diamond strength no chip nail polish" $\left(1.276 \pm 0.025 \mathrm{~g} / \mathrm{cm}^{3}\right)$ was determined. To ensure that the extraction process employed successfully removed any polish residue from the glitter particles and thus provided an accurate density measurement, we placed approximately 30 pieces of Alpha Jewels I glitter (1.394 \pm 0.002 $\mathrm{g} / \mathrm{cm}^{3}$ ) into $0.25 \mathrm{~g}$ of Sally Hansen diamond strength nail polish and repeated the extraction and collection process. We compared the levitation height (i.e., the density) of the Alpha Jewels before and after acetone extraction, and found the height of each sample to be within the standard deviation of the measurement. This method of extracting glitter from nail polish samples is, thus, an accurate means of obtaining density values from complex cosmetic matrices.

We determined the density of six smokeless gunpowder samples; the results are summarized in Figure 4b. We levitated each sample in $4.0 \mathrm{M} \mathrm{MnCl}_{2}$ (except for Hercules Red Dot, which we levitated in $3.0 \mathrm{M} \mathrm{MnCl}_{2}$ ) with 1.4500 and $1.8000 \mathrm{~g} / \mathrm{cm}^{3}$ density standard beads. We investigated the effect of repeated exposure and/or prolonged exposure to an aqueous $\mathrm{MnCl}_{2}$ solution, which are very acidic in nature with a $\mathrm{pH}$ of 3.0, on the density of gunpowder samples. We placed Hercules Blue Dot samples into a solution of $4.0 \mathrm{M} \mathrm{MnCl}_{2}$ and the density was measured every 24 hours for a total of seven days. The presence of density standards provided a means of accounting for evaporation of water from the solution, and thus changes in the solution density. The change in the average density of the gunpowder after seven days of exposure to aqueous $\mathrm{MnCl}_{2}$ was $0.012 \mathrm{~g} / \mathrm{cm}^{3}$, which is within the standard deviation of the initial measurements.

The density of Hercules Blue Dot gunpowder is also not affected by repeated exposures to $\mathrm{MnCl}_{2}$ solution. We levitated a sample of gunpowder for ten consecutive 
measurements, to determine if repeated exposure to $\mathrm{MnCl}_{2}$ causes changes in the density of the sample. We placed the gunpowder into $\mathrm{MnCl}_{2}$ solution, levitated it in the MagLev device, removed it from the $\mathrm{MnCl}_{2}$ solution, rinsed it with water, dried it with nitrogen, and repeated the process. The levitation height variation for each measurement was within the standard deviation determined in Figure $4 \mathrm{~b}$.

Density-based separation of mixtures with MagLev.

MagLev can also separate a mixture of objects by their density. We separated a mixture of glitter of similar size and shape (Mirror Crystalina I and Chrome Silver 1P), which were indistinguishable by eye as they have the same shape and size, however their relative thicknesses are easily determined with a light microscope (Figure 5a). A mixture of multiple densities is readily separated in the MagLev device (Figure 5b), this allows samples that are indistinguishable by eye (or by microscopy) to be separated. An added benefit of separation by MagLev is that samples can be readily sorted: a mixture is placed within the MagLev device, each sample reaches it appropriate levitation height based on density, the sample is removed from the magnet and each discreet sample is collected before the mixture recombines (e.g., glitter is readily removed from the MagLev solution with a pipette).

\section{Sources of Error in a MagLev Measurement.}

There are a number of errors that can result in an inaccurate density measurement; therefore care must be taken when performing a measurement with MagLev. Errors in solution preparation can result in measured density values that are higher or lower than expected. 
Incorrect density measurements can also arise if the concentration of the paramagnetic solution changes over time (e.g., by solvent evaporation). Density standards afford an absolute measure of density and reduce incorrect readings caused by variations in the concentration of ions in the paramagnetic solution. An added benefit of using density standards is that measurements made in different laboratories and under different experimental conditions (i.e., temperature, humidity, etc.) are comparable.

The presence of air bubbles on a sample results in a density measurement that is less than the true density value because air is much less dense than a typical sample. It is important to eliminate air bubbles from a sample before conducting a measurement. We measured the density of a glitter sample (Mirror Crystalina I) in cuvettes containing 3.0 $\mathrm{M} \mathrm{MnCl}$ solutions prepared under a variety of conditions: a $\mathrm{MnCl}_{2}$ solution, a $\mathrm{MnCl}_{2}$ solution containing $0.1 \%$ ( $\mathrm{vol} / \mathrm{vol}$ ) detergent (Tween 20 ), and a $\mathrm{MnCl}_{2}$ solution that was degassed. The glitter sample was added to each solution, inverted several times to thoroughly mix the glitter particles, inspected by eye for the presence of bubbles, and a density measurement taken. The average levitation height for each sample preparation was within the standard error of the other measurements (i.e., within $95 \%$ confidence, with $n=7$ measurements of 20 pieces of glitter per measurement).

We then sonicated each cuvette for 20 seconds to remove excess, and difficult to see, bubbles that had collected on the walls of the cuvette or the samples. In the absence of detergent, sonication of the samples resulted in a number of glitter particles adhering to the walls of the cuvette that were very difficult to remove. The spread of levitation heights of glitter within the cuvette was reduced when detergent was present and the sample was sonicated. Removing bubbles from samples is imperative to obtain an 
accurate density measurement. We found that adding a small amount of detergent (Tween $20,0.1 \%(\mathrm{vol} / \mathrm{vol}))$ and quickly sonicating the solution resulted in the most precise measurement of density for the glitter particles. The average density measurement obtained for each glitter sample was similar, independent of the solution conditions (e.g., the addition of detergent, sonication of the sample). The standard deviations for the glitter samples, however, were larger when detergent was not added and the sample was not sonicated.

The presence of tightly adhered particles (e.g., dust, grit, etc.) whose densities are different than the sample of interest will lead to an inaccurate density measurement. Careful preparation methods, in which the sample is cleaned but not altered, are needed to ensure that unwanted materials are removed from the sample. We have shown that the removal of nail polish from a glitter sample (via a simple extraction process with acetone and filtration step) does not alter its density. Similar preparation processes can be implemented for other samples. The practice of carefully preparing and analyzing samples of interest is an integral part of forensic science and quantitative analytical chemical methods.

\section{Conclusions.}

MagLev is a convenient and low-cost means for accurately determining the density (with a resolution of $0.001 \mathrm{~g} / \mathrm{cm}^{3}$ ) of a diamagnetic object. The density of an object can be determined from its levitation height in the MagLev device. The density values obtained from a sample, when compared with known density standards, are not dependent on the MagLev device used. Thus, any forensic laboratory using MagLev 
could contribute to a generally available database and also access it for an estimate of how common or rare their sample might be.

An accurate density determination can aid in determining if a questioned sample is, in fact, different from a known sample. We have demonstrated that the density of trace objects, glitter particles and smokeless gunpowder, can be determined with the MagLev system. We have shown that repeated density measurements and prolonged storage of trace objects in aqueous $\mathrm{MnCl}_{2}$ does not affect their density. We show that trace objects found in commercial cosmetic products can be extracted and their densities analyzed. We show that density measurements can differentiate glitter particles that are similar in appearance. 


\section{References.}

[1] M. C. Grieve, Journal of the Forensic Science Society 1987, 27, 405.

[2] P. L. Kirk, Density and Refractive Index; their Application in Criminal Identification, Thomas, Springfield, IL, 1951.

[3] K. A. Mirica, S. S. Shevkoplyas, S. T. Phillips, M. Gupta, G. M. Whitesides, Journal of the American Chemical Society 2009, 131, 10049.

[4] K. A. Mirica, S. T. Phillips, C. R. Mace, G. M. Whitesides, Journal of Agricultural and Food Chemistry 2010, 58, 6565.

[5] K. A. Mirica, S. T. Phillips, S. S. Shevkoplyas, G. M. Whitesides, Journal of the American Chemical Society 2008, 130, 17678.

[6] U. Andres, Magnetohydrodynamic \& magnetohydrostatic Methods of Mineral Separation, John Wiley \& Sons, New York, NY, 1976.

[7] CRC Handbook of Chemistry and Physics, 91 ed., CRC Press, Cleveland, 2010.

[8] Rasband, W.S., ImageJ, U.S. National Institutes of Health, Bethesda, MD, USA, http://imagej.nih.gov/ij/, 1997-2011.

[9] R. D. Blackledge, J. Edwin L. Jones, in Forensic Analysis on the Cutting Edge: New Methods for Trace Evidence Analysis (Ed.: R. D. Blackledge), Wiley Interscience, Hoboken, NJ, 2007, pp. 1.

[10] K. Aardahl, S. Kirkowski, R. D. Blackledge, Science and Justice 2005, 45, 7.

[11] Meadowbrook Inventions Inc., Bernardsville, NJ, USA, http://www.meadowbrookinventions.com, 2011.

[12] R. M. Heramb, B. R. McCord, Forensic Science Communications 2002, 4.

[13] E. O. Espinoza, J. I. Thornton, Analytica Chimica Acta 1994, 288, 57.

[14] W. J. Tilstone, K. A. Savage, L. A. Clark, Encyclopedia of Forensic Science: An Encyclopedia of History, Methods, and Techniques, ABC-CLIO, Inc., Santa Barbara, CA, 2006.

[15] W. Moorehead, in Forensic Analysis on the Cutting Edge: New Methods for Trace Evidence Analysis (Ed.: R. D. Blackledge), Wiley Interscience, Hoboken, NJ, 2007.

[16] An excess of glitter was used to prepare Figure 2 and 3, for easier visualization. These figures are not representative of an actual experiment.

[17] D. A. Skoog, J. F. Holler, T. A. Nieman, Principles of Instrumental Analysis, 5 ed., Saunders College Publishing, Fort Worth, TX, 1998.

[18] S. Griggs, J. Hahn, H. K. S. Bonner, Global Forensic Science Magazine 2011, 1, 19. 

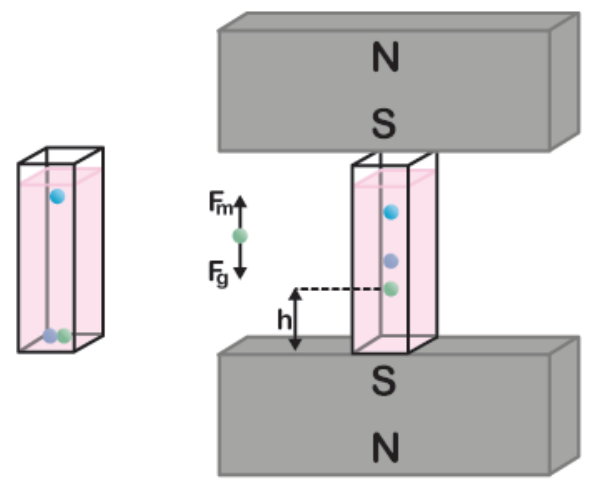

Figure 1. Diamagnetic objects are placed in a paramagnetic solution, $\mathrm{MnCl}_{2}$ in this work, and sink (purple and green spheres) or float (blue sphere) depending on the density of the object in comparison to that of the solution (left). The diamagnetic objects levitate, according to their density, when placed in a MagLev device that is composed of two magnets with like poles facing one another. The levitation height " $\mathrm{h}$ " of each object occurs when the gravitational force on the object $\left(\mathrm{F}_{\mathrm{g}}\right)$ is cancelled by the magnetic force $\left(\mathrm{F}_{\mathrm{m}}\right)$ applied in the opposite direction. 


\begin{tabular}{llll} 
Glitter Sample $^{a}$ & Thickness $(\mu \mathrm{m})$ & Size $(\mu \mathrm{m})$ & Shape \\
\hline${ }^{b}$ Alpha Jewels I & 25 & $204 \times 204$ & square \\
${ }^{b}$ Alpha Jewels II & 25 & 635 & hexagonal \\
${ }^{c}$ Alpha Jewels Epoxy & 50 & 635 & hexagonal \\
${ }^{d}$ Chrome Silver 1P & 178 & 635 & hexagonal \\
${ }^{e}$ Crystalina \#321 & $28-36$ & 635 & hexagonal \\
${ }^{e}$ Mirror Crystalina I & $28-36$ & 635 & hexagonal \\
${ }^{e}$ Mirror Crystalina II & $28-36$ & $204 \times 204$ & square \\
${ }^{d}$ Silver 1P Epoxy I & 25 & $102 \times 102$ & square \\
${ }^{d}$ Silver 1P Epoxy II & 178 & $102 \times 102$ & square \\
${ }^{d}$ Silver 1UP Ultrathin Polyester & 13 & 635 & hexagonal \\
${ }^{f}$ Silver Plastic Jewels \#21 & 178 & $380 \times 380$ & square
\end{tabular}

Table 1. Glitter samples that were analyzed with MagLev.

${ }^{a}$ The composition of each glitter particle, as reported from Meadowbrook Inventions,

Inc.

${ }^{b}$ Holographic glitter particles consisting of micro-embossed vacuum metalized $(0.5 \%$ aluminum) PET.

${ }^{c}$ Holographic glitter particles consisting of micro-embossed aluminum copolymer particles.

${ }^{d}$ Metallic glitter particles consisting of vacuum metalized ( $0.5 \%$ aluminum) pigmented PET.

${ }^{e}$ Iridescent glitter particles with a polyester/acrylic optical core and a polyester outer layer.

${ }^{f}$ Metallic glitter consisting of a copolymer. 


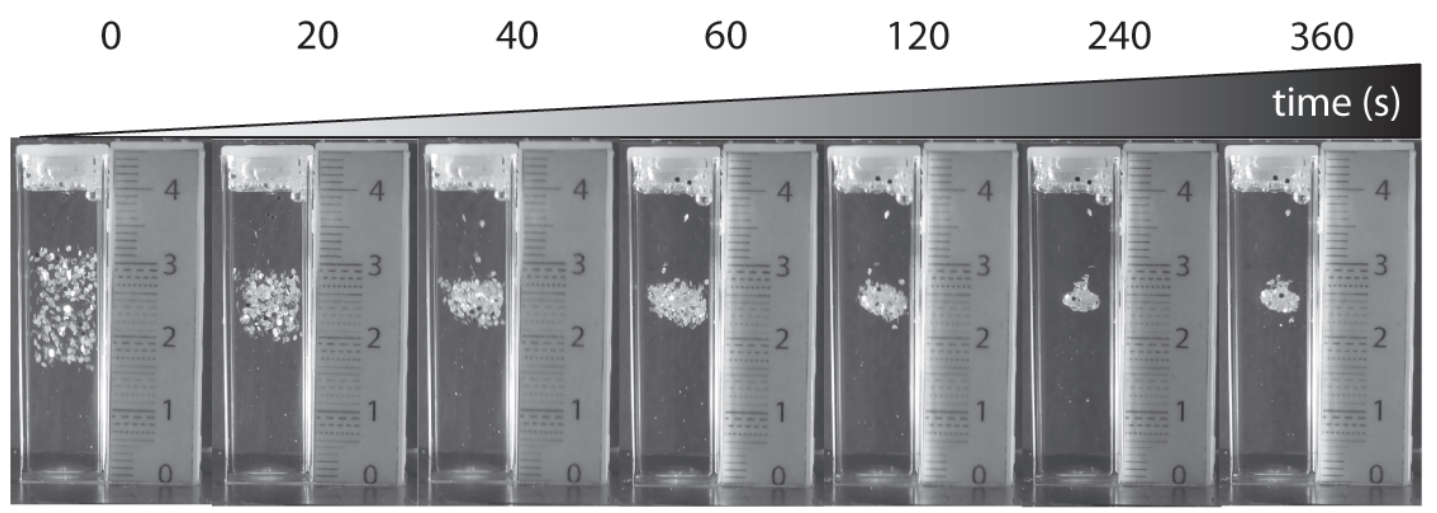

Figure 2. A sample of glitter (Mirror Crystalina I) was placed in cuvette containing an aqueous solution of $3.0 \mathrm{M} \mathrm{MnCl}_{2}$, shaken to disperse the glitter throughout the solution, and placed in the MagLev device. A series of time-lapse photographs were taken to show the time required for a large number of glitter particles to reach the appropriate levitation height of $2.5 \mathrm{~cm}$, determined by their density. A typical density measurement was performed with a smaller number of glitter particles than shown in these photographs; this large number of particles $(\sim 100)$ is for demonstration and ease of visualization. The objects above $4 \mathrm{~cm}$ are air bubbles at the top of the $\mathrm{MnCl}_{2}$ solution. 
a.

glitter particle \#

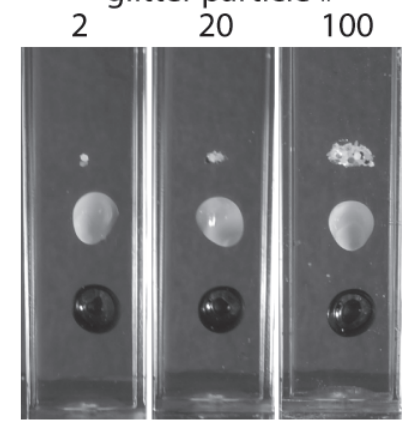

b.

\begin{tabular}{lcc} 
& reported density & measured density \\
\hline Alpha Jewels I & 2.4 & $1.394 \pm 0.002$ \\
Alpha Jewels II & 2.4 & $1.392 \pm 0.004$ \\
Alpha Jewels Epoxy & 1.4 & $1.385 \pm 0.001$ \\
Chrome Silver 1P & 1.4 & $1.389 \pm 0.010$ \\
Crystalina \#321 & 1.36 & $1.260 \pm 0.003$ \\
Mirror Crystalina I & 1.36 & $1.258 \pm 0.003$ \\
Mirror Crystalina II & 1.36 & $1.286 \pm 0.041$ \\
Silver 1P Epoxy I & 1.4 & $1.383 \pm 0.027$ \\
Silver 1P Epoxy II & 1.4 & $1.395 \pm 0.040$ \\
Silver 1UP Ultrathin Polyester & 1.4 & $1.394 \pm 0.012$ \\
Silver Plastic Jewels \#21 & 1.4 & $1.391 \pm 0.014$
\end{tabular}

Figure 3. (a) A sample of glitter (Crystalina \#321) was levitated in a cuvette containing 3.0 $\mathrm{M} \mathrm{MnCl}_{2}$ and two density standards $\left(1.350\right.$ and $1.450 \mathrm{~g} / \mathrm{cm}^{3}$; light and dark color, respectively). Each sample (containing 2, 20, or 100 pieces of glitter) was placed in the MagLev device and photos were taken after 360 seconds. (b) Density values for each glitter sample analyzed (measured density) and the density values reported by the manufacturer (reported density). The reported values are from seven independent measurements, with each measurement containing 20 pieces of glitter. 
a.

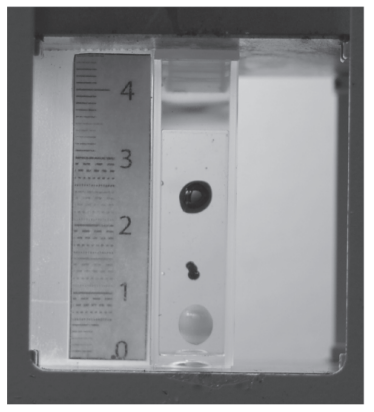

b.

height $(\mathrm{mm})$ density $\left(\mathrm{g} / \mathrm{cm}^{3}\right)$

$\begin{array}{lll}\text { Hercules Red Dot } & 27.5 \pm 0.28 & 1.226 \pm 0.010 \\ \text { IMR Trail Boss } & 18.9 \pm 0.74 & 1.557 \pm 0.059 \\ \text { Hercules Bullseye Orange } & 15.3 \pm 0.20 & 1.655 \pm 0.023 \\ \text { Hercules Blue Dot } & 14.8 \pm 0.13 & 1.657 \pm 0.015 \\ \text { IMR PB } & 13.2 \pm 0.28 & 1.660 \pm 0.030 \\ \text { IMR Hi Skor 800-X } & 13.1 \pm 0.31 & 1.662 \pm 0.025\end{array}$

Figure 4. (a) A sample of gunpowder (Hercules Blue Dot) was levitated in a cuvette containing a $4.0 \mathrm{M} \mathrm{MnCl}_{2}$ solution and two density standards $\left(1.450\right.$ and $1.800 \mathrm{~g} / \mathrm{cm}^{3}$; dark and light color, respectively) was placed in the MagLev device and a photo taken after 360 seconds. (b) The levitation height and corresponding density values for each gunpowder sample analyzed. The reported values are from seven independent measurements, with each measurement containing 3 pieces of gunpowder. 


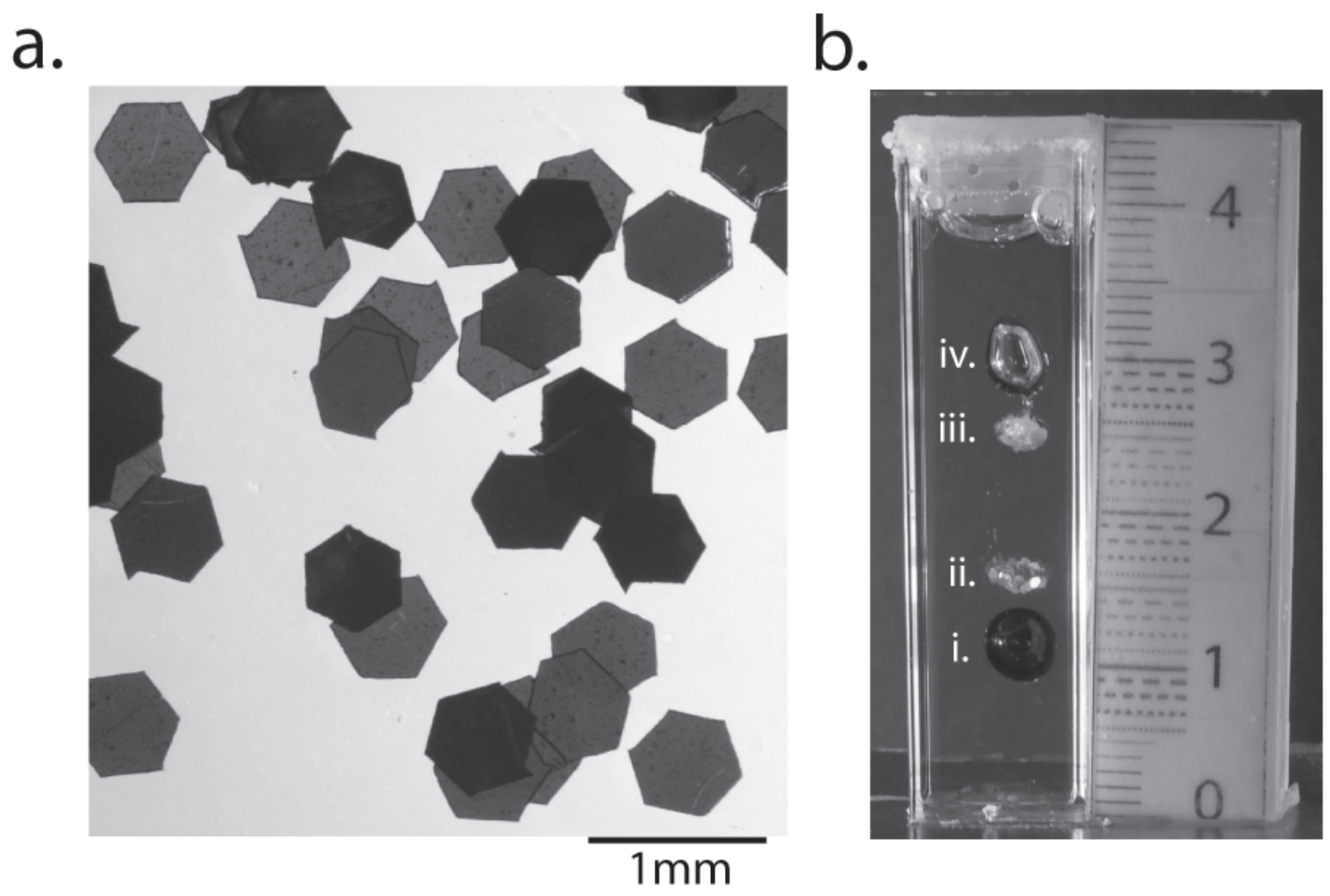

Figure 5. (a) An optical microscope image of a mixture of Mirror Crystalina I and Chrome Silver 1P glitter samples. The two glitter samples are difficult to distinguish without the aid of a microscope, as they are the same size and shape. Their relative darkness is representative of their differences in thickness. (b) The mixture was placed in a cuvette containing 3.0 $\mathrm{M} \mathrm{MnCl}_{2}$, placed in the MagLev device, and a photograph was taken after 360 seconds. The cuvette contains (i) a $1.450 \mathrm{~g} / \mathrm{cm}^{3}$ density standard, (ii) the sample of Chrome Silver 1P glitter, (iii) the sample of Mirror Crystalina I glitter, and (iv) a $1.350 \mathrm{~g} / \mathrm{cm}^{3}$ density standard. The mixture of glitter contained $\sim 50$ pieces of each glitter type. 